Revue d'histoire de l'Amérique française

ZRS REVUE D.HISTOIRE DE L'AMÉRIQUE FRANÇAISE

\title{
La thèse messianique et les Franco-Américains
}

\section{André Sénécal}

Volume 34, numéro 4, mars 1981

URI : https://id.erudit.org/iderudit/303904ar

DOI : https://doi.org/10.7202/303904ar

Aller au sommaire du numéro

Éditeur(s)

Institut d'histoire de l'Amérique française

ISSN

0035-2357 (imprimé)

1492-1383 (numérique)

Découvrir la revue

Citer cet article

Sénécal, A. (1981). La thèse messianique et les Franco-Américains. Revue

d'histoire de l'Amérique française, 34(4), 557-567.

https://doi.org/10.7202/303904ar d'utilisation que vous pouvez consulter en ligne.

https://apropos.erudit.org/fr/usagers/politique-dutilisation/ 


\title{
LA THÈSE MESSIANIQUE ET LES FRANCO-AMÉRICAINS
}

\author{
ANDRÉ SÉNÉCAL \\ Department of Romance Languages \\ The University of Vermont
}

Au problème fondamental du rôle de la société, de la culture et de l'économie québécoises dans le contexte d'une Amérique du Nord dominée par les éléments anglo-saxons, protestants et capitalistes, l'élite nationaliste du milieu du $19 \mathrm{e}$ siècle riposta en élaborant une thèse messianique. On proclama que l'unicité des moeurs, de.la langue et de la culture des descendants des Français dépendait de leur fidélité au catholicisme. À partir d'une telle interprétation des fondements d'une nation et d'une civilisation, on en vint à proclamer l'élection sacrée des héritiers de Champlain. À la veille de la Confédération, l'abbé Casgrain, qui empruntait ses mots à Rameau de Saint-Père, renchérissait sur l'avenir de la race:

Quelle action la Providence nous réserve-t-elle en Amérique? Quel rôle nous appelle-t-elle à y exercer? Représentants de la race latine, en face de l'élément anglo-saxon, dont l'expansion excessive, l'influence anormale doivent être balancées, de même qu'en Europe, pour le progrès de la civilisation, notre mission et celle des sociétés de même origine, éparses sur ce continent, est d'y mettre un contre-poids en réunissant nos forces, d'opposer au positivisme anglo-américain, à ses instincts matérialistes, à son égoïsme grossier, les tendances d'un ordre plus élevé qui sont l'apanage des races latines: une supériorité incontestée dans l'ordre moral et intellectuel, dans le domaine de la pensée. ${ }^{1}$

Malgré l'allusion de Casgrain à des «sociétés de même origine, éparses sur ce continent», les premiers prophètes du destin de la race latine en Amérique du Nord ne réservaient aucun rôle positif aux milliers des leurs qui s'acheminaient vers «les États». L'excom-

1 «Le mouvement littéraire au Canada», Oeuvres complètes (Montréal, Beauchemin, 1896), I: 369.

RHAF, vol. 34, no 4, mars 1981 
munication cinglante attribuée à George-Étienne Cartier, «Laissezles partir, c'est la canaille qui s'en va», est sans doute apocryphe. Elle ne traduit pas moins la prise de conscience angoissée de chefs politiques et religieux impuissants à détourner l'immigration vers les Saint-Jérôme et les Péribonka. En 1869, Le Nouveau Monde, l'organe des cohortes ultramontaines, lance des sentences fulgurantes contre l'émigré, cet être déchu «qui subit la juste peine de son apostasie», cette bête de somme qui devient par ses moeurs et ses tendances «l'être le plus méprisable de la société dans laquelle il vit» ${ }^{2}$.

Pourtant, la même année, Mgr de Goësbriand, évêque de Burlington, parle d'élus franco-américains «appelés de Dieu à coopérer à la conversion de l'Amérique comme leurs ancêtres furent appelés à planter la foi sur les bords du Saint-Laurent ${ }^{3}$. Au cours des années suivantes, des voix nationalistes respectées vinrent à partager la vision grandiose de l'évêque breton du Vermont. Dans son opuscule publié en 1880, Hier, aujourd'hui et demain, ou origines ei aestinées canadiennes, Charies Thibault of̂fre une version orthodoxe de cette réhabilitation:

Si l'exode de nos frères vers la république voisine n'était pas le voeu de la Providence, il serait plus qu'un crime, ce serait une faute irréparable au point de vue de nos forces, de notre grandeur, de nos richesses et de nos espérances nationales.

Nous nous devons ce tardif aveu, que nos frères des Etats-Unis nous valent sous tous rapports. ${ }^{4}$

Le texte de Thibault est un des premiers à proclamer la mission providentielle de la tribu perdue. Il s'ajoute à ceux de Ferdinand Gagnon et d'Hugo Dubuque qui, de bonne heure, avaient prédit la transformation de l'exode en cinquième colonne prête à confondre les forces de l'hérésie et du matérialisme dans leur principale redoute.

2 Cité par Gérard Bouchard, «Apogée et déclin de l'idéologie ultramontaine à travers le journal Le Nouveau Monde, 1867-1900», Fernand Dumont et al., Idéologies au Canada français, 1850-1900 (Québec, Presses de l'Université Laval, 1971), 124.

3 Ces mots sont tirés d'une lettre écrite en 1869 , que Le Protecteur Canadien de Saint-Albans (Vermont) publia dans son numéro du 11 mai 1869. Le texte fut repris dans Louis de Goësbriand, Les Canadiens des Etats-Unis (s.l., s. éd., s.d. [1889]). Nous citons de cette édition, 6 .

4 Charles Thibault, Hier, aujourd'hui et demain, ou origines et destinées canadiennes (Montréal, s. éd., 1880), 15. 
Du thème felix culpa, on passa vite à une interprétation plus exaltée de la mission du peuple de Dieu aux États-Unis, à un rêve que l'abbé Aristide Magnan allait qualifier de «trop beau» ${ }^{5}$. Les âmes les plus enthousiastes ne préconisèrent rien de moins qu'une terra franca qui annexerait le territoire des «Bastonnais» au foyer de la Nouvelle-France. Le jésuite Edouard Hamon se laissa séduire par cette vision. Dans Les Canadiens français de la NouvelleAngleterre, un ouvrage qui date de 1890, il laissa ses lecteurs entrevoir «le rêve trop beau»:

Que faut-il, en effet, pour que, dans un avenir plus ou moins rapproché, cette union s'opère [...]? Simplement ceci: que ces deux fractions d'une même race gardent fidèlement, chacune de son côté, certains éléments essentiels qui, le temps venu, puissent former un tout homogène, une véritable nationalité; c'est-à-dire, la religion, la langue et des traditions communes. En Canada, ces éléments sont désormais hors de tout danger, ils resteront. Dans les États-Unis de l'Est, [...] les 400,000 Canadiens émigrés font des efforts énergiques et heureux pour conserver, eux aussi, les mêmes avantages. Quand donc la réserve des Canadiens du Canada rejoindra les corps d'armée nombreux et déjà compacts, qui lui ont servi d'avant-garde aux États; quand ces hommes, issus d'une même race, parlant la même langue, adorant le même Dieu, se rencontreront face à face, à la frontière américaine, qui les empêchera de se reconnaitre pour frères, de se donner la main et de s'unir pour ne plus former qu'un seul et même peuple? ${ }^{6}$

Tout un corps de prophètes surenchérirent sur cette prédiction déjà ahurissante d'un avenir triomphant. Adélard Lemoine, par exemple, dans sa préface à $L$ 'Evolution de la race française en Amérique de Bruno Wilson, témoigne d'une foi des plus aveugles:

Sans les nôtres, sans cet exode providentiellement voulu, décrété, aurions-nous la gloire de compter là-bas [en NouvelleAngleterre] une population jalouse, comme nous, de conserver et sa langue, et sa religion, et sa foi? Verrions-nous là-bas comme une nouvelle terre canadienne en voie de réaliser cette prédiction d'un grand patriote [Edmond de Nevers], d'un voyant qui disait, avec la conviction d'un coeur d'apôtre, qu'un

5 Aristide Magnan, Histoire de la race française aux États-Unis (Paris, Charles Amat, 1912).

6 Edouard Hamon, Les Canadiens-français de la Nouvelle-Angleterre (Québec, N-S Hardy, 1891), 145-146. 
jour viendrait où le Mississipi serait la ligne de démarcation entre deux grandes races: «l'Est», disait-il, "sera canadienfrançais, l'Ouest sera plutôt anglais».'

À quoi attribuer un tel optimisme, cette volte-face fulgurante des interprètes du messianisme canadien-français? D'abord, aux proportions du flot migratoire. Certains affirment que, vers 1900 , on pouvait compter autant de Canadiens français hors du Québec qu'à l'intérieur. D'après les calculs de Ralph Vicero, on pouvait retrouver 40000 Canadiens français en Nouvelle-Angleterre vers 1860,575000 à la fin du siècle ${ }^{8}$. Loin d'être perdus à jamais pour les forces civilisatrices des Latins, comme l'avait prédit le camp ultramontain des années 1850-1860, les milliers d'émigrés s'empressèrent d'ériger des paroisses et de construire des écoles, de fonder des journaux et des sociétés culturelles, bref de mettre en place la même infrastructure qui avait permis à leurs pères de déjouer les plans des Durham et des Poulett-Thomson. Les premières paroisses canadiennes-françaises en Nouvelle-Angleterre datent des années 1850 et 1860 . Très tôt, les prêtres, les enseignants, les hommes de profession et les journalistes de combat vinrent rejoindre leurs ouailles, les ouvriers. Déjà, en 1890 , on pouvait compter 86 paroisses de langue française dans l'Est des Etats-Unis, et 50 écoles où l'enseignement était bilingue. Le flot migratoire du tournant du siècle permit aux orateurs de citer des chiffres encore plus impressionnants. En 1910, on relevait 202 paroisses et 101 missions desservies par 432 prêtres d'origine canadienne-française. Plus de 2000 frères et soeurs enseignaient la langue et la religion du Canada français à 55000 élèves. À la même époque, 3500 Franco-Américains poursuivaient leurs études dans les collèges classiques de la province de Québec.

Cet épanouissement inespéré encouragea les idéologues à méditer plus profondément sur les visées secrètes de la Providence et à

7 Adélard Lemoine, «Préface», Bruno Wilson, L'Évolution de la race française en Amérique (Montréal, s. éd., 1921), vol. I: ix.

8 La quantification de l'émigration canadienne-française aux États-Unis demeure un travail périlleux pour le statisticien. Pour une discussion du problème et une présentation de l'évidence, on consultera l'oeuvre de Yolande Lavoie, en particulier L'Émigration des Québécois aux États-Unis de 1840 à 1930 (Québec, Editeur officiel, 1979), et L'Émigration des Canadiens aux États-Unis avant 1930 (Montréal, Les Presses de l'Université de Montréal, 1972). Pour l'émigration en Nouvelle-Angleterre seulement (et avant 1900), on consultera avec profit la thèse de doctorat de Ralph Vicero, "Immigration of French Canadians to New England, 1840-1900: A Geographical Analysis», University of Wisconsin, 1968. Les chiffres que nous citons proviennent de Ralph Vicero, «L'Exode vers le Sud: Survol de la migration canadienne-française vers la Nouvelle-Angleterre au XIX ${ }^{\mathrm{e}}$ siècle», Vie Française, revue trimestrielle, (1980): 6. 
réexaminer le bien-fondé de leur défiance absolue du climat moral et du système politique des États-Unis. L'essor des FrancoAméricains contrastait singulièrement avec l'étouffement du souffle catholique et français au Manitoba et dans les autres provinces anglaises. Dès l'affaire Riel et à mesure que les événements montraient que les orangistes n'allaient jamais tolérer la transplantation et l'épanouissement de l'éthos québécois en dehors de la vallée du Saint-Laurent, les âmes nationalistes devinrent de plus en plus attentives au traitement accordé aux émigrés en Nouvelle-Angleterre. En dépit de moeurs que les curés jugeaient dépravées, malgré le climat matérialiste, la paroisse, la langue et les autres institutions de base de la survivance semblaient s'affermir de jour en jour au sein de l'Union américaine. Ces développements ne montraient-ils pas le doigt du Très-Haut? Plusieurs, comme Adélard Lemoine, n'en doutèrent plus:

Comment, en effet, expliquer cette puissance d'expansion de notre race $[\ldots .$.$] ? La plupart des auteurs qui ont écrit sur le sujet$ l'attribuent à des forces surnaturelles dont la Providence garde encore le secret, mais dont les effets successifs et toujours de mieux en mieux accentués ont, de fait, jusqu'ici, contribué à repousser de la Nouvelle-Angleterre les éléments sectaires de la primitive époque de la colonisation de cette contrée. ${ }^{9}$

Dès le tournant du siècle, pour certains, la question n'était plus celle du triomphe éventuel des Canadiens français dans l'Est des États-Unis mais bien celle des structures géopolitiques qui allaient réunir la nation. «La nation», «le pays», «le peuple», John Hare a déjà souligné l'imprécision de ces concepts dans la bouche des hommes politiques et des idéologues québécois du siècle dernier ${ }^{10}$. Dans son article, «Apogée et déclin de l'idéologie ultramontaine à travers le journal Le Nouveau Monde» ${ }^{11}$, Gérard Bouchard a bien précisé qu'aux yeux des ultramontains de 1890 ou de 1900 , la notion de nationalité ne découlait pas de structures géopolitiques, mais d'une essence spirituelle manifestée dans l'Histoire, qui révélait le jeu de la Providence dans le destin des peuples. La race et la nationalité ne coïncidaient donc pas avec les limites géographiques du Québec; le pays n'avait pas de frontières. On peut noter toute l'ambiguiité des notions de «pays» et de «nation» dans l'emploi qu'en fait le sénateur canadien Joseph Tassé:

\footnotetext{
9 Adélard Lemoine, op. cit., I: viii.

10 John Hare, «Pays, patrie, nation, les liens d'appartenance dans le monde moderne», Critère, 22 (été 1978): 211-234.

11 Gérard Bouchard, op. cit., 123-124.
} 
Si les deux branches de la famille canadienne [celle du Canada et celle des États-Unis] sont appelées à des destinées politiques différentes, il est du moins une union étroite que nous pouvons former, c'est l'union des coeurs, des volontés, des aspirations nationales [nous soulignons]. Cette union peut être indestructible comme l'idée qui l'inspire, car elle repose sur un sentiment qui résiste à l'action du temps, à l'éloignement, aux circonstances les plus adverses; elle repose, dis-je, sur cet amour sacré que ressent tout homme pour son pays. ${ }^{12}$

Ces mots, «aspirations nationales», «amour sacré [...] pour son pays», l'éditeur anti-annexioniste de La Minerve les adressait à un auditoire franco-américain de Boston.

Les porte-parole d'Ottawa n'étaient pas les seuls à couvrir d'ambiguïté des concepts comme «pays» et «nation». Tout en leur conseillant d'abandonner toute mentalité d'exilés, Henri Bourassa rappelait aux Franco-Américains qu'ils appartenaient à un peuple distinct et que leur tâche était de prolonger en Amérique l'effort de la France chrétienne. Il ajoutait:

Cette tâche, ne l'oublions pas, nous sommes seuls à pouvoir la remplir en Amérique. Les Canadiens français et les FrancoAméricains constituent le seul groupe important, le seul peuple de race et de langue françaises, en dehors de l'Europe. ${ }^{13}$

Chez d'autres nationalistes, les notions de pays et de peuple n'étaient pas moins sources d'intégration, mais elles évoquaient des frontières moins floues. Dans son texte messianique publié en 1896, L'Avenir du peuple canadien-français, Edmond de Nevers préconisa l'annexion du Canada aux Etats-Unis et la survivance des Canadiens français et des Franco-Américains dans un «état contractuel» qui n'exclurait «nullement les liens antérieurs et la nationalité»" ${ }^{14}$. De Nevers découpait l'immense territoire en créant une «petite Allemagne» dans le Nord central du continent et en octroyant le Québec et la Nouvelle-Angleterre aux descendants des Français. L'écrivain constatait que «les émigrés n'ont pas quitté la patrie, ils l'ont agrandie» ${ }^{15}$, et il concluait: 1883), 13

12 Joseph Taché, Aux Canadiens français émigrés (Ottawa, Imprimerie du Canada,

13 Henri Bourassa, La Langue, gardienne de la Foi (Montréal, Bibliothèque de l'Action française, 1918), 49.

14 Edmond de Nevers, L'Avenir du peuple canadien-français (Paris, Henri Jouve, 1896), 395.

15 Ibid, 435. 
Entre nos frères devenus citoyens américains et nous, une union plus intime qu'entre les Français de France, les Suisses et les Belges de langue française, devra régner, car, en outre de la communauté de la langue, de la religion et des souvenirs, qui nous lie, nous avons toute raison de croire qu'un même avenir politique nous attend. Nous ne sommes que momentanément séparés. ${ }^{16}$

Il est évident que les voix nationalistes perspicaces ne succombèrent jamais à de tels phantasmes et qu'elles surent découvrir l'envers du rêve. De tels observateurs mesuraient le progrès lent mais inéluctable des forces de l'assimilation. Plusieurs, comme les abbés Desrosiers et Fournet, s'appliquèrent à tempérer l'espoir mal fondé des âmes les plus enthousiastes. Dans La Race française en Amérique, les deux prêtres concluaient laconiquement:

On a voulu voir dans cet exode le commencement de la conquête prochaine de cette partie de l'Union américaine devenue sur certains points un prolongement du pays français du SaintLaurent. Ces prévisions sont assurément optimistes. ${ }^{17}$

Le "rêve trop beau» n'allait pas se réaliser. Le travail sourd des forces assimilatrices se révéla brutalement au grand jour vers la fin de la première grande guerre. La propagande raciste du gouvernement Wilson contre les «Huns» ne fut qu'un signe avantcoureur. Une fois la croisade terminée, on ne chercha pas à endiguer le courant d'intolérance qui s'abattit sur les groupes ethniques non-anglophones. Par exemple, dans tous les États de la NouvelleAngleterre, on présenta, et parfois on adopta, des projets de loi visant à proscrire toute langue autre que l'anglais dans l'enseignement public ou confessionnel. Ces attaques visaient surtout les écoles paroissiales franco-américaines. Au niveau national, une association de gouverneurs d'État se prononça pour la suppression des journaux en langue étrangère. Des organismes comme la National Education Association, l'Institut Carnegie, le New York Times se firent les champions du mouvement «un drapeau, une langue». Les campagnes de public safety furent accompagnées de menées fanatiques contre tout ce qui était «étranger» à l'éthos américain et à la langue anglaise.

16 Ibid, 392.

17 Louis-Joseph-Adélard Desrosiers et Fournet, La Race française en Amérique, 2e éd. augm. (Montréal, Beauchemin, 1911), 241. 
La flamme xénophobe couvait depuis longtemps. Tout le long de la deuxième moitié du $19 \mathrm{e}$ siècle, les knownothings avaient fait valoir les prétentions anglo-saxonnes et protestantes à une supériorité innée. Â la fin du siècle, les instincts les plus racistes fermentaient dans les écrits d'une intelligentsia qui s'était emparée des impostures pseudo-scientifiques de Gobineau pour alerter les siens contre la marée de nouveaux immigrants qui fuyaient les ghettos de la Russie, les quartiers fétides de Naples ou la Chine mandchoue. D'éminents savants et des chefs politiques et religieux pontifièrent sur l'infériorité raciale des Latins, des Juifs et des Orientaux. En 1890, on vilipenda les Franco-Américains du Massachusetts en les appelant "the Chinese of the Eastern States». Vers 1920 , cette méfiance à l'égard du «stock» non nordique restait une foi scientifique. Nombre d'ouvrages, en particulier, The Passing of the Great Race (1916) de Madison Grant, le rapport de l'Institut Carnegie (1923) rédigé par Harry Laughlin, «expert eugenic agent», ainsi que le volumineux rapport de la commission fédérale Dillingham (1910-1923) conclurent que les derniers venus, les Latins, les Slaves, les Chinois, etc., sapaient le système démocratique, ruinaient l'entreprise capitaliste et semaient la violence, la prostitution et la maladie mentale.

$\mathrm{Au}$ niveau national, les forces qui tentèrent d'extirper la langue et la culture des derniers immigrants furent secondées par l'épiscopat irlandais. L'assemblée consultative des évêques, la National Catholic Welfare Conference, apaisa ceux qui attaquaient les écoles confessionnelles en leur assurant que «la politique éducationnelle catholique est d'insister pour que tous les sujets, sans excepter la religion, soient enseignés en anglais». La Conference eut beau ajouter: «La politique de l'Église, en cette matière, n'est pas de précipiter la solution du problème ${ }^{18}{ }^{18}$, elle ne put dissimuler les sympathies de certains de ses membres. Mgr Rice de Burlington, au Vermont, s'insurgea contre les députés de son État qui voulaient proscrire le français dans les écoles paroissiales, mais le rôle de Mgr Hickey du Rhode Island dans l'affaire du projet de loi Peck (le projet visait à extirper les langues étrangères des écoles) fut moins édifiant. Encouragé par les Hickey et les Walsh,

18 Traduction d'un extrait de $A$ Catechism of Catholic Education de la National Catholic Welfare Conference (1922) cité dans $L a$ Vocation de la race française en Amérique du Nord (Québec, Le Comité permanent de la survivance française en Amérique, 1945), 191. 
le clergé irlandais, qui retenait mieux le catéchisme de Tammany Hall que les leçons de l'évangile, s'évertua à étouffer tout ce qui retardait l'américanisation du catholicisme.

Dès le tournant du siècle, la curie romaine avait adopté une politique américaine (et canadienne-anglaise) qui préconisait l'assimilation de toute ethnie catholique sous l'égide de l'épiscopat irlandais. Dans le cas des Polonais, Rome alla jusqu'à accepter le schisme plutôt que de tempérer le zèle assimilateur de ses acolytes. Malgré les énoncés catégoriques du Vatican, des défaites et des victoires qui ne laissaient aucun doute quant à l'issue du combat (les affaires de Danielson, de North Brookfield, de Biddeford, etc.), les partisans irréductibles de la mission providentielle de la race canadienne-française en Nouvelle-Angleterre ne capitulèrent pas. Pendant longtemps, l'élite franco-américaine récusa les voix qui s'élevèrent pour prôner les bienfaits de l'assimilation et de l'hégémonie gaélique. Les prophètes de l'inévitable eurent peu de disciples avant l'affaire de La Sentinelle (1925-1929), une longue confrontation entre les évêques et les chefs franco-américains les plus imbus de la mission providentielle de leur race. À la fin, entre le schisme et la subordination des aspirations nationales à la realpolitik romaine, la majorité des patriotes, tant au Québec qu'en Nouvelle-Angleterre, se joignirent à Henri Bourassa pour condamner un nationalisme qu'on qualifia «d'outrancier». C'était sonner l'olifant.

L'affaire de La Sentinelle fut le reductio ad absurdum du nationalisme ultramontain québécois. À la suite de la faillite de l'esprit libéral et laïque de 1837 , le camp ultramontain s'était approprié la thèse nationaliste en imposant son interprétation théocratique et messianique du fait québécois. L'Église comprit très vite qu'un fossé linguistique s'avérait le rempart le plus formidable contre l'hérésie protestante. Face au zèle agressif de sectes et d'organismes comme la French Canadian Missionary Society, les chefs religieux en vinrent à proclamer un lien indissoluble entre langue et religion. Les idéologues ultramontains cimentèrent le lien étroit entre langue française et religion catholique en retenant ces deux attributs comme titre d'élection et de définition intrinsèque de la «nation» canadienne-française. En 1922, J.-Albert Foisy admettait que la foi ne dépendait pas de la langue pour vivre mais il ajoutait aussitôt que l'abandon de la langue maternelle créait une condition qui rendait la conservation de la foi catholique impossible «à moins d'un miracle». Foisy ajoutait: 
D'abord, la foi, tout en demeurant un don surnaturel, s'appuie, pour vivre, sur les traditions familiales et nationales, sur les pratiques religieuses et a besoin de l'atmosphère catholique. Tout cela suppose la conservation de la langue maternelle au moins dans la famille, dans l'Église et dans l'enseignement. Un individu ne perd sa langue que le jour où il a perdu la mentalité nationale, les traditions, les coutumes et les goûts de ses pères. ${ }^{19}$

À titre d'exemple du lien étroit entre religion et nationalité, entre religion et langue, Foisy cite le cas franco-américain:

D'un autre côté, toujours aux États-Unis, prenons le groupe des Canadiens français. Là où ils se sont américanisés, où ils ont perdu la langue comme leurs frères les Irlandais, ils se sont détachés de l'Église catholique. Partout où l'organisation paroissiale leur a permis de rester français de langue et de tradition, ils sont tous catholiques. ${ }^{20}$

Le dogme politico-religieux des ultramontains reposait sur un paradoxe fondamental puisque, de toujours, l'autorité suprôme do l'Église était antinationaliste.

L'affaire de La Sentinelle offrit une démonstration irréfutable de la politique romaine. Le Saint-Siège ne voulut voir dans le conflit qu'un cas d'insurbordination à l'autorité absolue de l'épiscopat. Les défenseurs de la thèse messianique, tant au Québec qu'en Nouvelle-Angleterre, durent accepter la décision de la curie et décourager toute résistance à la politique assimilatrice poursuivie par l'épiscopat américain. Les développements qui s'ensuivirent ne furent pas sans équivoque. Ainsi, il était inéluctable qu'à cause de son attachement aveugle à Rome, Henri Bourassa, l'auteur de La Langue, gardienne de la Foi, le patriote qui avait confondu Mgr Bourne, ne vît dans les sentinellistes que des révoltés, et dans leur combat pour leur langue et leur culture, qu'un «nationalisme outrancier».

Suite à cette affaire retentissante, de nombreux porte-parole s'empressèrent de prévenir les fidèles contre des thèses vicieuses. Citant «un éminent homme de sciences», l'abbé Hormisdas Hamelin, qui avait oeuvré de longues années auprès des FrancoAméricains, tira la conclusion inévitable: «Ceux qui s’imaginent

19 J.-Albert Foisy, La Langue maternelle (Montréal, Bibliothèque de l'Action Française, 1922), 11 .

20 Ibid, 7. 
que la race et la langue sont les éléments constitutifs de la nation versent dans un dangereux sophisme. ${ }^{21}$ Le prélat mit les points sur les «i» en ajoutant qu'il était incontestable «que la religion passe avant la nationalité» et qu'il était faux, pour les FrancoAméricains, de dire «Qui perd sa langue, perd sa religion». Sans broncher, l'abbé Hamelin conclut:

C'est une erreur pleine de dangers et capable des pires conséquences. Faites entrer cette idée-là dans la tête des gens et vous les amènerez à croire que s'ils ne peuvent rester français, ils ne sont pas tenus de rester catholiques $[\ldots]^{22}$

La majorité des chefs québécois joignirent leurs voeux à ceux de l'abbé Hamelin et, d'un trait, ils abandonnèrent les FrancoAméricains à leur avenir glorieux:

Ayons confiance en la divine Providence, et laissons à ses soins les Franco-Américains, sachant qu'elle répond merveilleusement à tous les besoins, nous rappelant «que ses jugements sont incompréhensibles, et ses voies impénétrables», qu'elle sait tirer le bien du mal, et qu'elle fait servir chaque individu, comme chaque groupe et chaque nationalité, à la réalisation du plan du divin Architecte, arrêté de toute éternité. ${ }^{23}$

Le concert de voix messianiques se tut. Les apôtres francoaméricains les plus récalcitrants se replièrent derrière la doctrine figée du Comité permanent de la survivance française en Amérique. L'ouvrage publié par le Comité en 1945, La Vocation de la race française en Amérique du Nord, reste le dernier coup de trompette nostalgique, le défi chimérique des champions irréductibles de cette vocation messianique.

21 Hormisdas Hamelin, Lettres à mon ami sur la patrie, la langue, et la question franco-américaine (s.l., s.éd., 1930), 220.

22 Ibid, 224.

23 Ibid, 255. 